\title{
Neuroevolutionary Multiobjective Optimization of Injection Stretch Blow Molding Process in the Blowing Phase
}

\author{
Renê S. Pinto ${ }^{1}$ Hugo M. Silva ${ }^{1}$, Fernando M. Duarte ${ }^{1}$, João P. Nunes ${ }^{1}$, \\ António Gaspar-Cunha ${ }^{1}$
}

\begin{abstract}
Injection stretch blow molding is a very important thermoplastic processing technique producing hollow containers with mechanical performance. One of the main challenges in optimizing this process consists in finding the best thickness profile for each part in order to achieve the desired mechanical properties with less material use. In a previous study, a new methodology based on a neuroevolutionary multiobjective optimization approach was proposed to enhance the entire process, which considers that the process is optimized by phases, starting by the end. In that initial study only the final phase of the process was addressed, where the best thickness profile for an industrial bottle was found in order to satisfy the required mechanical properties with less material use. In the present study, the focus is the second stage of the optimization methodology, concerning the blowing phase of injection blow molding process. The optimal results obtained in the first phase are used as the optimal thickness profile for the bottle with the goal to find the best preform thickness profile which produces the desired bottle. The same procedures are used and the results shown that the methodology was successfully applied to its second phase.
\end{abstract}

\section{Introduction}

Injection stretch blow molding is one of the most important processes in the industry to produce hollow plastic containers, such as bottles, jars and several kind of different hollow plastic parts. Basically, this thermoplastic processing technique comprises the following steps: 1) injection of molten raw material into a cavity to produce the desired shape of the preform; ii) heating the preform, typically by radiation, so that the material acquires deformation capability; iii) stretch and blowing the heated preform in order to ensure that the preform reproduces the contours of the mold. The stretch, made mechanically by the action of a plug, and the blowing,

\footnotetext{
${ }^{1}$ Institute of Polymers and Composites, University of Minho, Campus de Azurém, 4800-058 Guimarães, Portugal.
} 
using air under pressure, can occur sequentially (stretch followed by blowing) or at the same time; iv) finally, the part is cooled and removed from the mold.

Since the amount of material used in blow molded products represents a significant share of the total manufacture costs, the minimization of material utilization is required [1]. However, there are several important mechanical properties which are also dependent on this feature. Numerical approaches can be applied to avoid empirical tests to find the process input variables which gives the best tradeoff between the material utilization and the desired mechanical properties. Several studies in the literature present different approaches concerning injection stretch blow molding design process and optimization $[1,2,3,4,5,6]$. One of the major challenges in optimizing this process is to define the complete thickness profile and shape of the final part and of the preform in order to achieve desired mechanical properties with less material utilization.

In [1] and [2] a global optimization methodology for injection stretch blow molding process is presented and detailed. This methodology uses a neuroevolutionary multiobjective approach and is composed by steps (or phases) that should be performed to optimize the whole process in order to find the best thickness profile of the final part and of the preform. In both studies only the first phase of the optimization, which comprises the final stage of manufacturing process, is addressed. This study focuses on the second phase of the optimization methodology, which comprises the blowing of a stretched preform in the manufacturing process. In the previous study, optimal thickness distributions of the final part were obtained. The main goal of this study is find the best thickness distributions of the preform that will leads to final parts (after the blowing phase) with the optimal thickness profiles found on the previous study.

\section{Global optimization methodology}

The proposed optimization methodology for the injection stretch blow molding summarizes the whole process in five main phases: Injection, Stretching, Blowing, Mold opening and Blow-molded part. The injection phase comprises the melting of raw material and its injection into a cavity to form the preform. The stretching phase, that is not always present in the manufacturing process, comprises the stretching of the preform in order to maximize the amount of material at the bottom of the final part. The blowing phase comprises the injection of air under pressure to expand the preform towards the mold, acquiring its shape. Mold opening comprises the phase where the mold is opening and the final part is pulled out. Finally, Blowmolded part comprises the last production phase, where the final part is cooled and becomes ready for packaging.

After summarize the five main phases in the blow molding process, the optimization methodology establishes four phases (or steps) for the optimization process ( $\mathrm{O} 1$ to $\mathrm{O} 4)$. However, the optimization starts by the last production phase, i.e., when the final part is done. The first optimization step (O1) comprises in 
optimize the thickness profile of the final part, i.e., find the best thickness profile of the final part which provides the desired mechanical properties with less material utilization. Step O2 comprises in optimizing the preform thickness profile after stretching, i.e., find the thickness profile of the preform which will produce (after blowing) the final part with the optimal profile found in step O1. This study concerns on this phase. The step $\mathrm{O} 3$ comprises in the optimization of the preform thickness profile before stretch, i.e., finding the thickness profile of the preform (before stretch) that will produce (after stretch) the preform with the optimal profile found in step O2. Finally, the step O4 also optimizes the preform thickness profile, but injection conditions and cavity geometry are used as decision variables.

\subsection{Neuroevolutionary multiobjective optimization}

One of the insights of the proposed methodology is treat a container's thickness distribution as a function of its geometry. In this context, Artificial Neural Networks (ANNs) are built to compute the wall thickness at any location of the part (based on the corresponding 3D coordinate). To allow many evaluations throughout the optimization process, simulations are carried out through finite element models (FEMs). Thus, by using ANNs the search space can be drastically reduced once each FEM model is composed by a 3D mesh with thousands or even millions of points. In the evolutionary algorithm, each solution is represented by an ANN which gives a thickness distribution profile for a given FEM (3D mesh). The attributes of the ANN are evolved to find the networks that give optimal distributions. Fig. 1 illustrates the ANN representation.

Finite element model
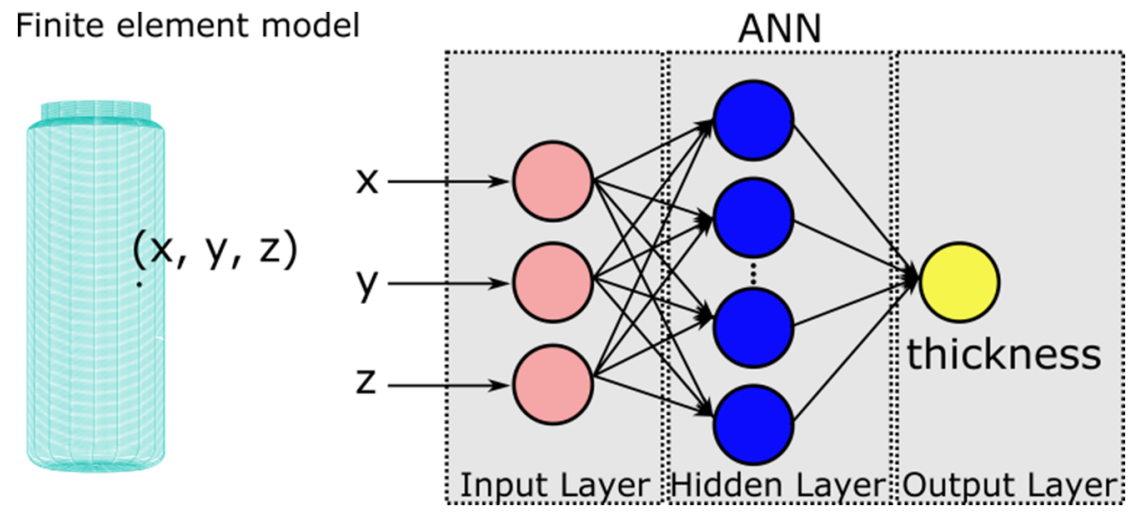

Fig. 1. A FEM model (bottle) mesh. Each coordinate of the mesh is an input to the ANN to calculate the thickness in the corresponding point.

The multiobjective optimization evolutionary algorithm of the methodology is based on the SMS-EMOA [7]. Fig. 2 illustrates the basic workflow for the optimization. 


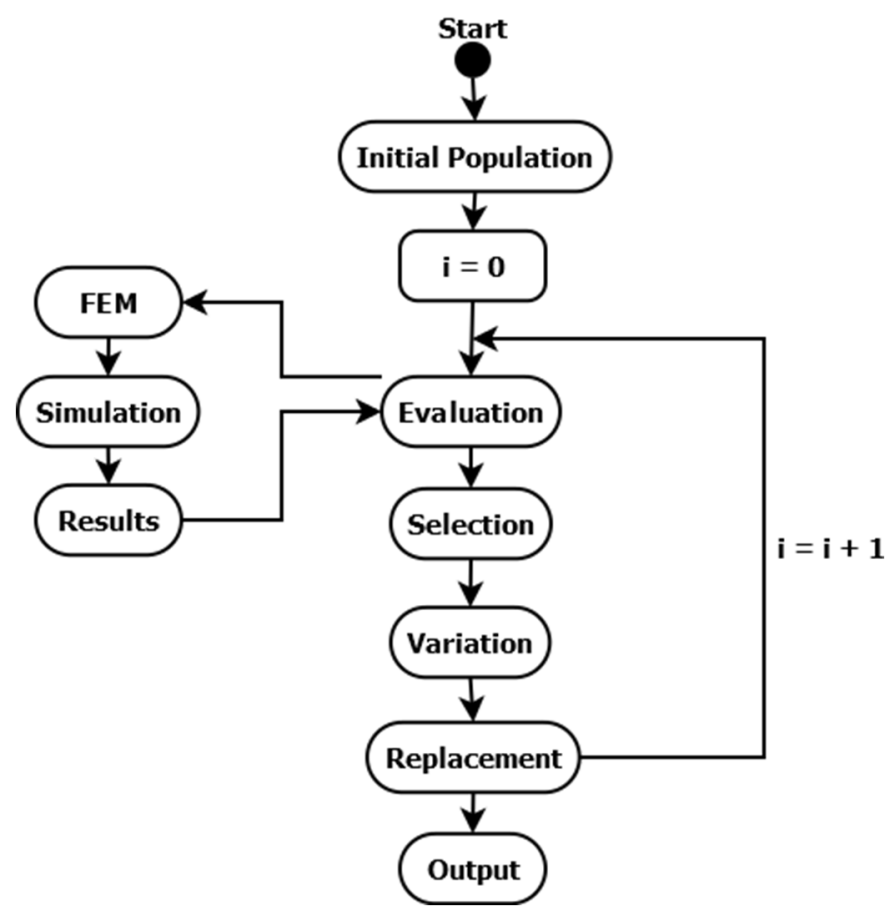

Fig. 2. Neuroevolutionary optimization methodology workflow

Each population is composed by a set of individuals (solutions), each one representing an ANN. The weights and biases of the ANN are encoded in a real number chromosome. Thus, the size of the chromosome depends of the ANN topology instead of the size of FEM model mesh. The initial population is generated randomly.

To evaluate a solution, the coordinates of each point of a given mesh are feed into the ANN to define the thickness in each one of the points, forming the thickness profile that is considered as the input by the simulation process. In the evolutionary algorithm, selection is performed by a uniform distribution and variation is performed by the SBX-Crossover operator, which is designed to work with real number representations. Replacement strategy is based on Pareto front and hypervolume [8] measure. As a result of the optimization process, there will be a set of optimal solutions where each of them represents an ANN that gives the wall thickness distribution for the model mesh. All solutions will provide different tradeoffs between the considered objectives, such as mass versus mechanical properties, for instance. 


\section{Experimental design}

In the first phase of the optimization methodology an industrial bottle model was considered in the experiments. The bottle is $45 \mathrm{~mm}$ in diameter and $182 \mathrm{~mm}$ in height, composed by plastic material with mass density of $1.15 \mathrm{~g} / \mathrm{cm}^{3}$ and Poison's ratio of 0.4. The applied air (blowing) pressure and Young's module ratio is 0.0027 . Fig. 3 shows the 3D mesh model with dimensions indicated. The vertical lines of the mesh are highlighted to illustrate the points where the wall thickness is calculated.

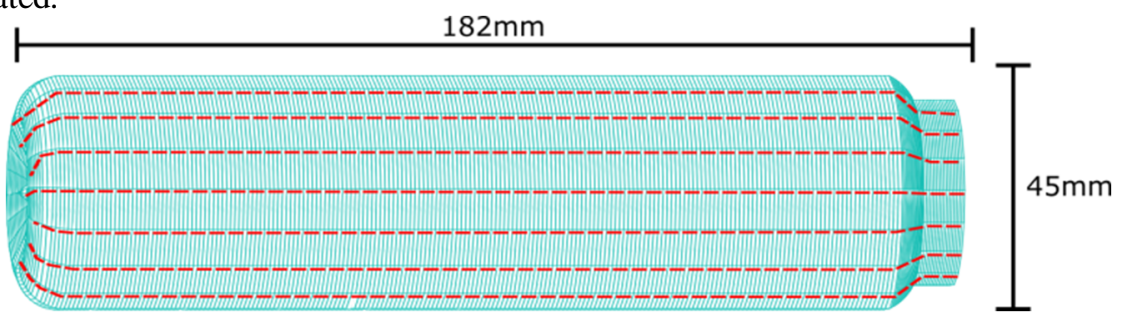

Fig. 3. Bottle model with vertical lines of the mesh highlighted

In the first phase three objective functions were considered to be minimized: $f_{1}$, the total mass of the bottle; $f_{2}$, the maximum strain suffered by the bottle and $f_{3}$, which is the maximum difference between the thickness profile of all vertical lines, measured by RMSE index (root mean square error). This objective measures how uniform is the thickness distribution, since the same thickness profile for all vertical lines, i.e., along the bottle, is desirable.

Fig. 4 and Fig. 5 show the Pareto front of the final population for the first phase of the optimization. In Fig. 4 it can be seen that all solutions have low value for RMSE error (all below 0.01), which means that the algorithm was able to find uniform distributions. In Fig. 5 only the objectives $f_{1}$ and $f_{2}$ are plotted. All solutions are well distributed along the Pareto curve, providing different tradeoffs between the total mass $\left(f_{1}\right)$ and the maximum strain $\left(f_{2}\right)$. Five optimal solutions (S1 to S5) are highlighted in the curve. Solutions S2 and S3, which are located in the knee area, are considered to give the best (balanced) relationship between $f_{1}$ and $f_{2}$ objectives. Thus, they were considered as the optimal designs to be achieved by the second optimization phase on this study. 


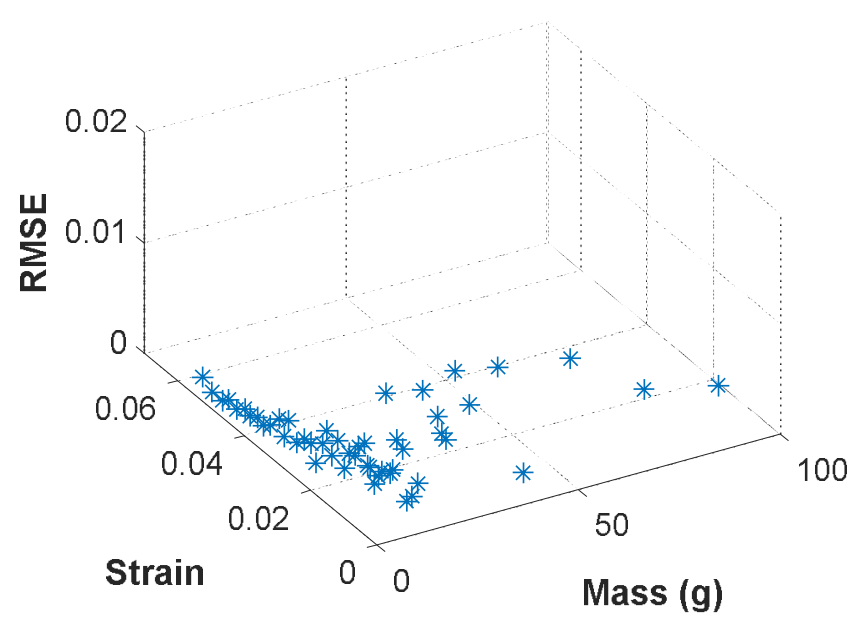

Fig. 4. Pareto front for final population for the first phase of the optimization process.

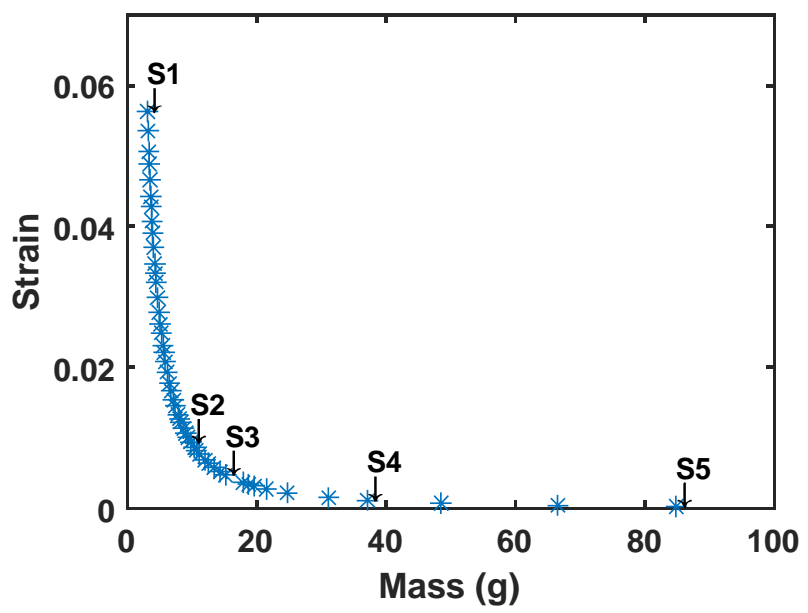

Fig. 5. Pareto front of the first phase with only objective functions $f_{1}$ and $f_{2}$ plotted.

Fig. 6 shows the thickness distribution for the optimal solutions S2 and S3. The $\mathrm{x}$-axis comprises the points located from the bottleneck towards the bottom of the bottle. Each line (distribution) represents the mean thickness values between all vertical lines of the mesh (Fig. 3). Both distributions presented the same behavior. Concerning solution S2, all the points presented mean thickness values of $0.30 \mathrm{~mm}$ which decreases faster up to $0.03 \mathrm{~mm}$ when reaches the bottom of the bottle. Solution S3 had the same behavior, but the mean thickness value was $0.58 \mathrm{~mm}$ decreasing up to $0.06 \mathrm{~mm}$ at the bottom. From a physical point of view, these results 
make sense. In S2, the bottle wall is thinner, using less material, but it suffers more strain than solution S3, where the wall is thicker, using more material, but it suffers lower maximum strain. Tab. 1 lists $f_{1}$ (total mass) and $f_{2}$ (maximum strain) values for both solutions.

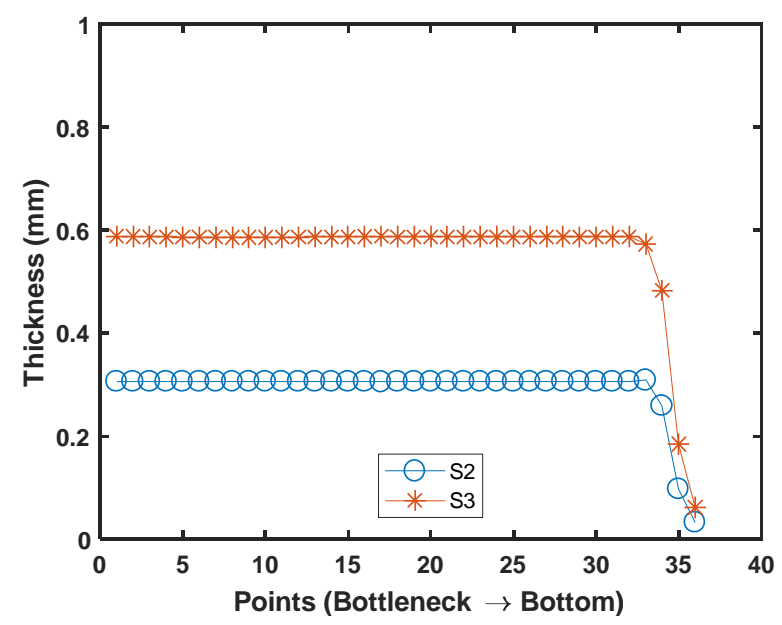

Fig. 6. Thickness distribution of optimal solutions S2 and S3

Table 1. Total mass and maximum strain values for solutions S2 and S3

\begin{tabular}{ccc}
\hline Solution & Total Mass (g) & Maximum Strain $\left(\mathbf{x 1 0 ^ { - 3 } )}\right.$ \\
\hline S2 & 9.8 & 9.4 \\
S3 & 15.2 & 4.8 \\
\hline
\end{tabular}

Once the optimal thickness distributions for the final part (bottle) were obtained in the first phase of the optimization, the second phase comprises in find the best thickness profile of the preform, before the blowing phase, that will produce the final part (with optimal thickness profile) after blowing procedure. To compare the thickness distribution of the final part (after blowing) with an optimal thickness distribution, two objectives were defined for the second phase:

$$
\begin{aligned}
& f_{1}=\frac{1}{M} \sum_{i=1}^{M} \frac{\left|y_{i}-\widehat{y}_{i}\right|}{y_{i}} \\
& f_{2}=\max _{1 \leq i \leq M} \frac{\left|y_{i}-\widehat{y}_{l}\right|}{y_{i}}
\end{aligned}
$$


Where $y_{1}, y_{2}, \ldots, y_{M}$ comprise the mean thickness value for each point along all vertical lines in the final part (after blowing) and $\hat{y}_{1}, \hat{y}_{2}, \ldots, \hat{y}_{M}$ comprise the mean thickness value for the corresponding points in the optimal (or target) distribution, such as in $\mathrm{S} 2$ or S3. Thus, $f_{1}$ is the mean error between the distributions and $f_{2}$ is the maximum error.

A preform 3D mesh model was designed to produce the same bottle model used in the first phase throughout a blow molding simulation using ANSYS Workbench software. An initial population composed by a set of ANNs that provide preform thickness profiles were randomly generated and evolved through the optimization algorithm. The same parameters (number of individuals per population, number of generations and network topology) from the first phase were used.

Two experiments (Exp1 and Exp2) were carried out: in Exp1 the optimal solution S2 (from first phase) was considered as the optimal (target) thickness profile to be reached in the final part (after the blowing procedure). In Exp2, the optimal solution S3 was considered as the optimal profile.

\section{Results and discussion}

Figure 7 shows the evolution of the hypervolume on each generation for Exp1 and Exp2 (normalized values). It can be seen that both experiments presented higher hypervolume on its final populations, evidencing the evolution of each population throughout the optimization process.

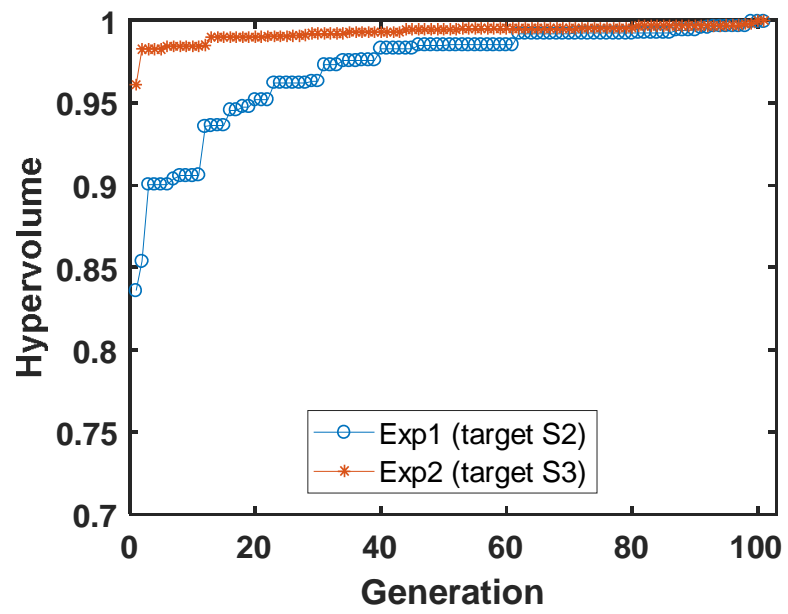

Fig. 7. Evolution of the hypervolume on each generation for Exp1 and Exp2

Fig. 8 and Fig. 9 emphases the optimization process by showing the initial and final population for Exp1 and Exp2, respectively. 
Fig. 10 and Fig. 11 show the Pareto front (all non-dominated solutions) of final population for Exp1 and Exp2. An optimal solution was manually selected in each curve taking into account the most balanced relationship between the two objectives. In Exp1, solutions are spread across the curve while in Exp 2 solutions are concentrate between 0.055 and 0.06 on the x-axis. Also, a lower number of nondominated solutions were found when comparing with Exp1.

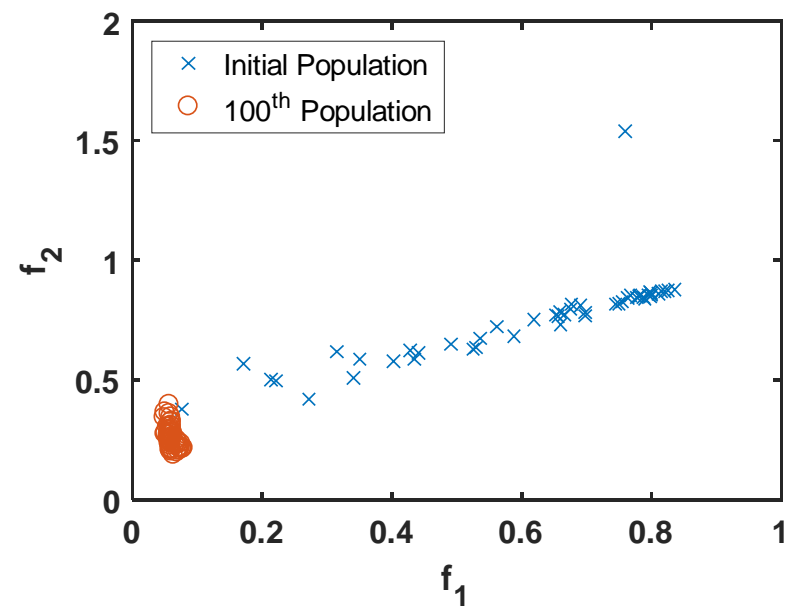

Fig. 8. Initial and final population for Exp 1

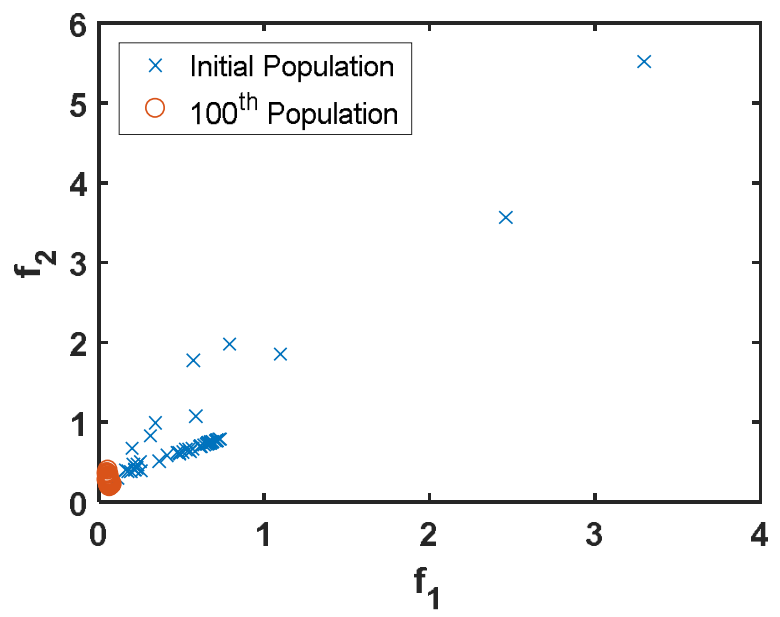

Fig. 9. Initial and final population for Exp 2 


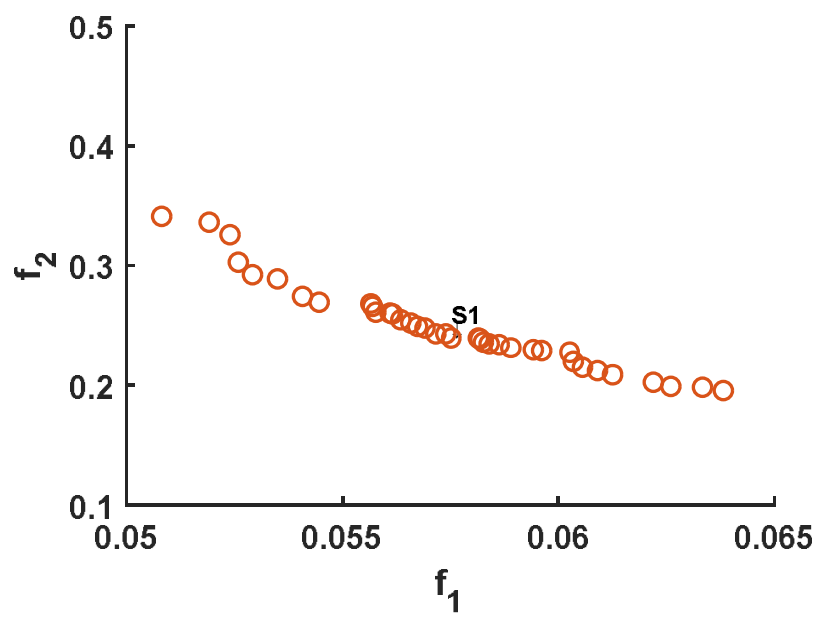

Fig. 10. Pareto front of Exp 1. Optimal solution S1 is highlighted

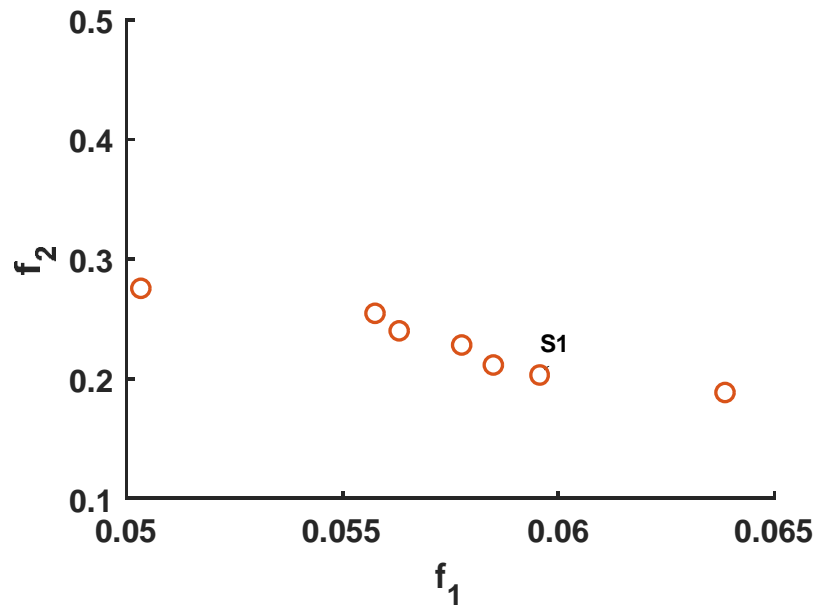

Fig. 11. Pareto front of Exp 2. Optimal solution $S 1$ is highlighted

Fig. 12 and Fig. 13 show the thickness distribution for the optimal solutions selected from Exp 1 and Exp 2. The corresponding target distribution, i.e., the optimal distribution found in the first phase, is also presented on each graph. Tab. 2 lists the numerical values for objective functions of both solutions. 


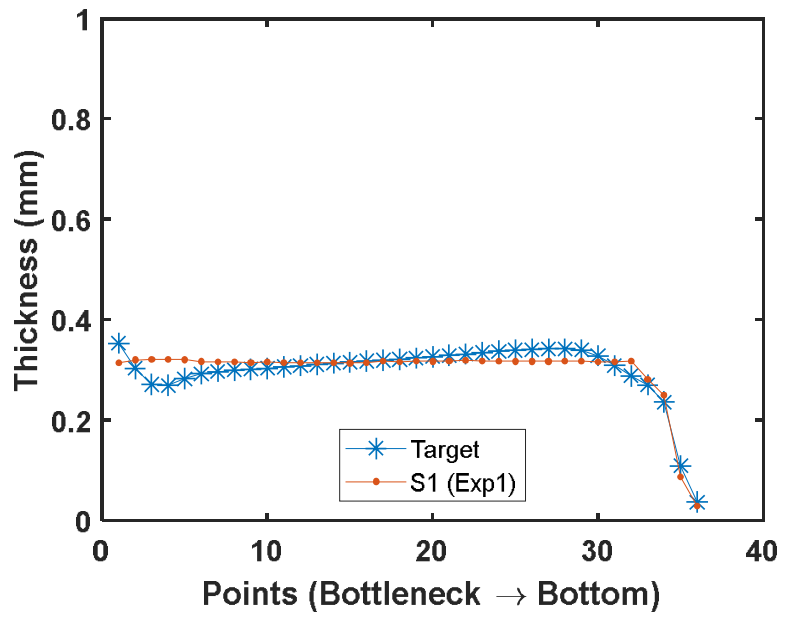

Fig. 12. Thickness distribution of solution $S 1$ from Exp1 (target distribution is $S 2$ from the first phase)

Table 2. Objective function values for optimal solutions selected from Exp1 and Exp2

\begin{tabular}{ccc}
\hline Solution & $\mathbf{f}_{1}$ & $\mathbf{f}_{2}$ \\
\hline S1 - Exp1 & 0.0575 & 0.0596 \\
S1 - Exp2 & 0.2386 & 0.2026 \\
\hline
\end{tabular}

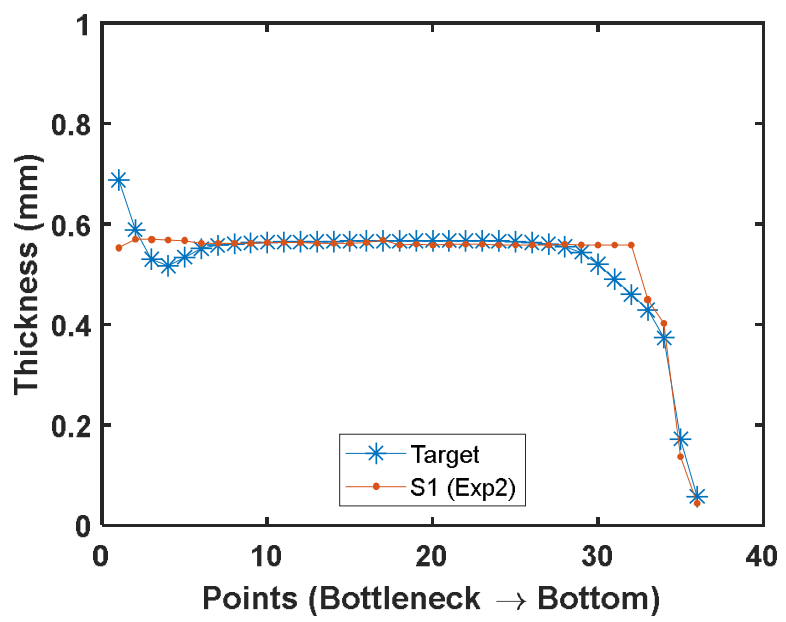


Fig. 13. Thickness distribution of solution $S 1$ from Exp2 (target distribution is $S 3$ from the first phase)

Both solutions presented mean error $\left(f_{1}\right)$ of order 0.05 . Concerning the precision generally involved in the manufacturing process, this error is irrelevant, which means that the thickness profiles found for the preform will produce the final bottle with the desired thickness distribution after the blowing process.

Concerning the maximum error $\left(f_{2}\right)$, the values obtained were 0.2386 and 0.2026 for Exp1 and Exp2, respectively. Although it represents around 20\% of error, it is important to point out that $f_{2}$ is a single point with the highest divergence between the resulted and the target thickness. For a mean thickness distribution of $0.3 \mathrm{~mm}, 20 \%$ represents $0.06 \mathrm{~mm}$, which is also very small concerning the manufacturing process.

\section{Conclusions}

Injection stretch blow molding is a process widely used by the industry to produce hollow plastic parts. The optimization of this process can heavily decrease production costs by finding thickness profiles which gives best tradeoffs between different objectives, such as material utilization and mechanical properties. Previous studies had proposed a neuroevolutionary multiobjective optimization methodology for this process. The methodology is divided in four phases (or steps), but only the first step was previously covered. This study addressed the second phase of the methodology, which corresponds to the optimization of the blowing phase in the manufacturing process.

Using the optimal thickness profile of the final part found in the first phase of the optimization process, the second phase performed the optimization with the goal to find the preform thickness profile that produces the final part with the optimal profile after the blowing process. Two optimal profiles from the first phase were considered and the same procedures for optimization were followed, defining the appropriate objective functions and FEM models for the current phase. The results comprise a set of solutions that provide different thickness distributions for the preform (through ANNs) that will produce the final part with the desired optimal profile with a mean error of 5\%, which is irrelevant considering the precision of manufacturing process. Two optimal preform thickness profiles were selected from the Pareto front and can be used in the next phase of the optimization process.

Future works should address the other steps of the optimization methodology. The next phase of the optimization should consider the optimal preform profiles found on this study.

Acknowledgments This work has been supported by the European project MSCA-RISE-2015, NEWEX, with reference 734205. 


\section{References}

1 R. Denysiuk, N. Gonçalves, R. S. Pinto, H. Silva, F. M. Duarte, J. P. Nunes and A. GasparCunha, "Optimization of Injection Stretch Blow Molding: Part I - Defining Part Thickness Profile," International Polymer Processing, vol. XXXIV, 2019.

2 R. Pinto, H. Silva, F. Duarte, J. Nunes and A. Gaspar-Cunha, "Neuroevolutionary Multiobjective Methodology for the Optimization of the Injection Blow Molding Process," in International Conference on Evolutionary Multi-Criterion Optimization, 2019.

3 R. Denysiuk, F. M. Duarte, J. P. Nunes and A. Gaspar-Cunha, "Evolving neural networks to optimize material usage in blow molded containers," EUROGEN - International Conference on Evolutionary and Deterministic Methods for Design Optimization and Control with Applications to Industrial and Societal Problems, 2017.

4 J. Biglione, Y. Béreaux, J.-Y. Charmeau, J. Balcaen and S. Chhay, "Numerical simulation and optimization of the injection blow molding of polypropylene bottles-a single stage process," International Journal of Material Forming, vol. 9, pp. 471-487, 2016.

5 C. Hopmann, S. Rasche and C. Windeck, "Simulative design and process optimization of the two-stage stretch-blow molding process," in AIP Conference Proceedings, 2015.

6 G.-Q. Huang and H.-X. Huang, "Optimizing parison thickness for extrusion blow molding by hybrid method," Journal of Materials Processing Technology, vol. 182, pp. 512-518, 2007.

7 N. Beume, B. Naujoks and M. Emmerich, "SMS-EMOA: Multiobjective selection based on dominated hypervolume," European Journal of Operational Research, vol. 181, pp. 1653$1669,2007$.

8 E. Zitzler and L. Thiele, "Multiobjective optimization using evolutionary algorithms-a comparative case study," in international conference on parallel problem solving from nature, 1998. 